\title{
PÉRDIDA AUDITIVA INDUCIDA POR RUIDO EVALUADA EN ODONTÓLOGOS DOCENTES DE LAS CLÍNICAS ODONTOLÓGICAS DE LA UNIVERSIDAD SANTO TOMAS
}

\author{
${ }^{1}$ Yinna LIzzeth Rivera B., ${ }^{2}$ Sandra Juliana Rueda $V{ }^{3}$ Sonia Constanza Concha S. \\ ${ }^{1}$ Estudiante X Semestre, F. de Odontología, U. Santo Tomas, Fonoaudióloga U. Manuela Beltrán. \\ ${ }^{2}$ Odontóloga, U. Santo Tomá. Especialista en Patología Oral y Medios Diagnósticos, U. El Bosque, Docente U. Santo Tomás \\ ${ }^{3}$ Odontóloga, U. Santo Tomás, Especialista en Educación y Comunicación para la Salud, \\ Magíster en Epidemiología, U. Industrial de Santander, Docente U. Santo Tomás.
}

\section{Autor responsable de la correspondencia: Yinna Lizzeth Rivera B.}

Correo electrónico: lizzeth20@hotmail.com

\section{RESUMEN}

Objetivo: Identificar el grado de pérdida auditiva inducida por ruido (PAIR) en odontólogos docentes de las clínicas de la Universidad Santo Tomás según la calificación auditiva de las escalas ocupacionales de Larsen y ELI.

Materiales y Métodos: Se realizó un estudio de tipo observacional descriptivo de prevalencia. El universo lo constituyeron 32 odontólogos; la muestra fue de 27 odontólogos seleccionados mediante muestreo voluntario. Se analizó la relación de la presencia de PAIR con cada variable mediante test de $\mathrm{Chi}^{2}$, $t$ de Student y test exacto de Fisher; se consideró un nivel de significancia $\alpha=0.05$. Como variables de salida la presencia de pérdida auditiva según ELI y Larsen para ambos oídos y como variables explicatorias las relacionadas con los aspectos sociodemográficos y clínicos.

Resultados: La prevalencia de PAIR según la calificación de la escala ELI fue en el oído izquierdo en un 62,9\%; las mujeres evidenciaron alteración más marcada a este nivel. En la escala Larsen, el oído derecho reportó el 88,9\% de prevalencia siendo los hombres quienes presentaron mayor alteración.

Conclusión: Según la valoración de la escala ELI, las mujeres evidenciaron el inicio de la alteración auditiva en la frecuencia de 4000 $\mathrm{Hz}$ y en los hombres hubo compromiso de las frecuencias agudas y conversacionales siendo notoria la evolución de la pérdida auditiva. [Rivera YL, Concha SC. Perdida auditiva inducida por ruido evaluada en odontólogos docentes de las clínicas odontológicas de la Universidad Santo Tomas. Revista Ustasalud Odontología 2007; 6: 96 - 103]

Palabras Clave: Pérdida auditiva, Audiometría, Larsen, ELI, Ruido, Riesgo Profesional.

\section{LOST HEARING INDUCED BY NOISE EVALUATED IN EDUCATIONAL DENTISTS IN THE DENTAL CLINICS IN SANTO TOMAS UNIVERSITY}

\section{ABSTRACT}

Objective: To identify the degree of auditory loss in educational dentist of the Santo Tomas University dental clinics according to the audiology qualification of the occupational scales of Larsen and ELI.

Materials and Methods: A observational descriptive study of prevalence was made; the universe was 32 dentists and the sample was 27 dentists, selected by voluntary sampling. The relation of the presence of PAIR with each variable was analyzed by Chi ${ }^{2}$, Student's $t$ and Fisher Exact test; a significance level was considered $\alpha=0.05$. The exit variables were the presence of loss audition according to ELI and Larsen for both ears and like explanations variables the related ones to the sociodemographics and clinical aspects.

Results: The prevalence of PAIR according to the qualification of the ELI scale was in the left ear in a 62.9\%; the women demonstrated noticeable alteration at this level. In the scale Larsen the right ear report $88.9 \%$ of prevalence being the men who presented/displayed greater alteration.

Conclusion: According to the valuation of the ELI scale the women demonstrate the beginning of the auditory alteration in the frequency of 4000 Hertz and in the men there is commitment of the acute and conversational frequencies being well-known the lost evolution of the auditory one.

Key words: Hearing loss, Eudiometry, Larsen, ELI, Noise, Professional risk.

Recibido para publicación: 7 de julio de 2007. Aceptado para publicación: 4 de febrero de 2008.

* Grupo de Investigación Riesgos Profesionales. 


\section{INTRODUCCIÓN}

El oído como órgano sensorial es fundamental en la audición y el equilibrio. Esta dividido en tres partes con funciones importantes y especificas como son: el oído externo, oído medio y el oído interno. ${ }^{1}$

La pérdida auditiva inducida por ruido es progresiva, el límite de exposición estipulado y permitido por el National Institute for Ocupational Safety and Health (NIOSH) debe ser de 85 decibeles menos de 8 horas diarias, si se expone a $90 \mathrm{~dB}$ máximo 8 horas y de $95 \mathrm{~dB}$ sólo 4 horas. Al estar expuesto al ruido, el profesional se acostumbra a éste sin ser conciente del peligro irreparable al que esta sometiendo su audición y, generalmente, evade este factor de riesgo por desconocimiento real del tema., ${ }^{2,3}$

El profesional de la odontología es susceptible a presentar problemas de audición relacionados con su desempeño laboral al estar en contacto directo con factores de riesgo entre los cuales esta el ruido producido por la pieza de alta velocidad, los succionadores de aire, la lámpara de fotocurado, el compresor, el aire acondicionado entre otros. ${ }^{2}$ Además, es necesario tener en cuenta la intensidad y la duración del ruido durante la jornada laboral de 8 horas con respecto a la distancia del oído y la fuente generadora del riesgo. ${ }^{4}$

Souza encontró en sus investigaciones que las diferentes marcas de turbinas incrementaban la cantidad de decibeles; ${ }^{5}$ además, sustentó que la posición de la pieza de alta dentro de la boca puede generar mayor o menor sonido siendo el área mas susceptible la zona anterior de la cavidad oral, no obstante aborda la posición del odontólogo con respecto al paciente pues entre mas cerca este de él, más posibilidad tendrá la onda sonora de recorrer menor distancia al oído del operador, esta sustentación también fue apoyada por Patrik, en 1981. ${ }^{5}$

El odontólogo en algún momento de su ejercicio profesional experimenta y refiere síntomas relacionados con el ruido debido a la exposición diaria a éste entre las cuales se presenta disminución de la agudeza auditiva, tinitus, migraña, irritabilidad, estrés, falta de concentración, atención entre otros ${ }^{5,6}$, generando un efecto negativo al interferir en el desempeño de las labores en el consultorio odontológico o en su vida cotidiana.

Para llevar a cabo la valoración auditiva teniendo en cuenta el diagnostico netamente ocupacional es fundamental aplicar en los resultados del audiogra- ma las escalas de calificación Larsen y ELI que son tenidas en cuenta para determinar si un trabajador se encuentra afectado por el riesgo físico que implica el ruido dentro de su ambiente laboral. ${ }^{6,7}$

La escala ELI determina el índice de pérdida auditiva temprana (trauma acústico), se obtiene restándole al umbral audiométrico observado en la frecuencia de $4.000 \mathrm{~Hz}$ el respectivo factor corrector de presbiacusia basado en la edad y el sexo. ${ }^{7}$ La escala Larsen modificado es una medida auditiva según criterio ocupacional que denomina la hipoacusia neurosensorial grado I, II sin compromiso vía ósea, y aérea conversacional grado III con compromiso del área del lenguaje, definida como sensibilidad de la agudeza auditiva menor a $25 \mathrm{~dB}$ en todas las frecuencias. ${ }^{7}$

Cabe mencionar que es el primer trabajo de investigación que se realiza en odontólogos en la ciudad de Bucaramanga relacionando la pérdida auditiva inducida por ruido como riesgo profesional, lo cual conlleva a la necesidad de profundizar mas en el tema para priorizar el diseño de programas de promoción y prevención del fenómeno en estudio.

El propósito de esta investigación es identificar el grado de perdida auditiva según escalas de medición ocupacional en odontólogos docentes de las clínicas de la Universidad Santo Tomás debido al ruido al cual se exponen en su ambiente de trabajo.

\section{MATERIALES Y MÉTODOS}

Se desarrolló un estudio observacional descriptivo de prevalencia, ${ }^{8}$ el universo se constituyó por 32 odontólogos docentes de las clínicas de la Universidad Santo Tomás; de acuerdo con los criterios de exclusión, se recolectó una muestra de 27 odontólogos con características especificas para el estudio y que no tenían antecedentes médicos de origen hereditario que influyeran en el diagnóstico auditivo ocupacional.

Se incluyeron en el estudio odontólogos docentes activos de las clínicas de la Universidad Santo Tomás durante el 2006 con edades entre 32 y 70 años o con experiencia laboral entre 5 y 30 años, que trabajaran aproximadamente seis horas diarias sin protectores auditivos, que tuvieran consultorio particular y factores asociados a alteraciones auditivas no producidas por exposición a ruido, síntomas relacionados con alteración auditiva como otalgia, tinitus, dificultad para oír una conversación. 
Se excluyeron docentes con alteraciones auditivas hereditarias.

En esta investigación se aplicó un instrumento tipo anamnesis audiológica y audiograma para diagnóstico auditivo con criterios de calificación ocupacional determinados por la aplicación de las escalas Larsen modificado y ELI. Las variables que se consideraron fueron clasificadas en sociodemográficas como edad, género, estrato socioeconómico y el lugar de procedencia; adicionalmente se recolectaron variables clínicas como las diagnostico ocupacional a nivel auditivo como la escala Larsen, la escala ELI, el tiempo de exposición a ruido, factores asociados a perdida auditiva (golpes en la cabeza o cirugía de cabeza y oídos) y síntomas auditivos inducidos por ruido (zumbidos, otalgia, dificultad para oír una conversación).

Con la finalidad de evaluar el instrumento utilizado para recoger la información, se realizó una prueba piloto que mejoró la precisión y la pertinencia de las variables, para evitar errores durante la aplicación del estudio definitivo. La prueba fue efectuada en diez odontólogos particulares de la ciudad de Bucaramanga con características similares a la población objeto de estudio, a partir de ésta se modificaron criterios del instrumento, se estandarizó el procedimiento y se definió el tiempo para la prueba.

Para la recolección de la información se informó y explicó detalladamente a los pacientes el objetivo del estudio y el procedimiento del examen, se solicitó el consentimiento informado y se obtuvo la información requerida a través de la aplicación de la anamnesis. Cada odontólogo fue evaluado por la fonoaudióloga quien realizó las audiometrías laborales dentro de la cabina sonoamortiguada con previo reposo auditivo de 8 horas. Se evaluaron las frecuencias graves $(250 \mathrm{~Hz})$, las conversacionales (500- 1000 y $2000 \mathrm{~Hz}$ ) y las agudas (3000- 4000-6000 y $8000 \mathrm{~Hz}$ ). El diagnostico se estableció según las escalas ocupacionales ELI y Larsen en cada oído, con base en estos resultados se determinó si existía algún tipo de alteración y a que nivel.

La base de datos se elaboró en Excel $^{9}$ por duplicado y mediante la rutina Validate del paquete EPIINFO 6.04 se verificó la calidad de la digitación; ${ }^{10}$ finalmente, se exportó al paquete estadístico STATA 9.1 para el correspondiente procesamiento. ${ }^{11} \mathrm{El}$ plan de análisis estadístico univariado incluyó el cálculo de las medidas de resumen para las variables cualitativas como proporciones y para las variables cuantitativas medidas de dispersión y de tendencia central. ${ }^{12,13}$
En el análisis bivariado se analizó y evaluó la asociación de la variable pérdida auditiva según las escalas de calificación ELI y Larsen modificado con cada una de las variables explicatorias, se aplicaron los test $\mathrm{Chi}^{2}$, test Exacto de Fisher para variables cualitativas y prueba $t$ Student o test de rangos de Wilcoxon para variables cuantitativas dependiendo de la distribución de los datos. ${ }^{12,13}$ Para todo el análisis se considero un nivel de $\alpha=0.05$.

Las consideraciones éticas del estudio se acogieron a los principios fundamentados en la resolución 008430 del 4 de octubre de 1993 del Ministerio de Protección Social de la República de Colombia en los que se preservaron los principios de autonomía, eficiencia y justicia. El estudio de investigación registró un riesgo mínimo según el Artículo 11 Parágrafo B pues se examinó el umbral auditivo del odontólogo docente y se determinó el diagnóstico a nivel ocupacional lo que implicó el más bajo riesgo para la salud física y auditiva del paciente. ${ }^{14}$

\section{RESULTADOS}

\section{Análisis Univariado}

\section{Variables sociodemográficas}

Se evaluaron en total 27 odontólogos docentes de las clínicas de la Universidad Santo Tomás con una edad promedio de 48,4 \pm 11,7 años; el 59,3\% (16) eran hombres, el 100\% de los odontólogos evaluados de ambos sexos procedían del área urbana y el $55,6 \%$ pertenecía a estrato socioeconómico alto (Tabla1).

Recordando que la escala de medición y diagnóstico ocupacional ELI evalúa la frecuencia de $4000 \mathrm{~Hz}$ y aplica la correspondiente corrección por presbiacusia para determinar la calificación del trauma acústico se encontró que la prevalencia de pérdida auditiva en el oído derecho fue del $55,6 \%$ (15) y en el oído izquierdo fue del $62,9 \%$ (17). En las mujeres evaluadas, el $63,6 \%$ tuvo pérdida auditiva en el oído derecho y el $72,7 \%$ (8) en el oído izquierdo. Por otro lado para el estrato alto se observó pérdida auditiva bilateral del $73 \%$ (11). No se observaron asociaciones estadísticamente significativas de acuerdo a lo establecido en la escala ELI en oído derecho e izquierdo para ninguna de estas variables (Tabla1).

Recordando que la escala de calificación Larsen modificado mide el umbral auditivo y determina la 
Tabla 1. Descripción de las variables sociodemográficas analizadas en forma global y según la calificación de Trauma Acústico mediante la Escala ELI.

\begin{tabular}{|c|c|c|c|c|c|}
\hline \multirow{4}{*}{ VARIABLE } & \multicolumn{5}{|c|}{ TRAUMA ACÚSTICO } \\
\hline & GLOBAL & DERECHO & \multirow{3}{*}{$\mathbf{P}$} & IZQUIERDO & \multirow{3}{*}{$\mathbf{P}$} \\
\hline & \multirow{2}{*}{ Frec. (\%) } & PERDIDA & & PERDIDA & \\
\hline & & Frec. (\%) & & Frec. (\%) & \\
\hline Global & $27(100)$ & $15(55.6)$ & & $17(62.9)$ & \\
\hline Edad $\dagger$ & $48.4 \pm 11.71$ & $47.4 \pm 9.2$ & ${ }^{*} 0.6141$ & $47.41 \pm 11.71$ & ${ }^{*} 0.5606$ \\
\hline \multicolumn{6}{|l|}{ Género } \\
\hline Hombres & $16(59.3)$ & $8(50.0)$ & ${ }^{* *} 0.696$ & $9(56.3)$ & ${ }^{* *} 0.448$ \\
\hline Mujeres & $11(40.7)$ & $7(63.6)$ & & $8(72.7)$ & \\
\hline \multicolumn{6}{|l|}{ Procedencia } \\
\hline Urbano & $27(100)$ & $15(55.6)$ & & $17(63.0)$ & \\
\hline \multicolumn{6}{|l|}{ Estrato } \\
\hline Alto & $15(55.6)$ & $11(73.3)$ & ${ }^{* *} 0.057$ & $11(73.3)$ & ${ }^{* *} 0.257$ \\
\hline Medio & $12(44.4)$ & $4(26.7)$ & & $6(50.0)$ & \\
\hline$\alpha=0,05$ & ${ }^{*}$ Test de Student & & t exacto de & & $\dagger \mathrm{X} \pm \mathrm{DE}$ \\
\hline
\end{tabular}

Tabla 2. Descripción de las variables sociodemográficas analizadas en forma global y según el Umbral Auditivo Evaluado mediante Escala LARSEN.

\begin{tabular}{|c|c|c|c|c|c|}
\hline \multirow{4}{*}{ VARIABLE } & \multicolumn{5}{|c|}{ UMBRAL AUDITIVO (LARSEN) } \\
\hline & GLOBAL & DERECHO & \multirow{3}{*}{$\mathbf{P}$} & IZQUIERDO & \multirow{3}{*}{$\mathbf{P}$} \\
\hline & \multirow{2}{*}{ Frec. (\%) } & PERDIDA & & PERDIDA & \\
\hline & & Frec. (\%) & & Frec. (\%) & \\
\hline Global & $27(100)$ & $24(88.9)$ & & $23(85.2)$ & \\
\hline $\begin{array}{l}\text { Edad } \dagger \\
\text { Género }\end{array}$ & $48.4 \pm 11.71$ & $49.6 \pm 11.7$ & ${ }^{*} 0.1415$ & $49.5 \pm 12.0$ & ${ }^{*} 0.2597$ \\
\hline Hombres & $16(59.3)$ & $15(97.8)$ & ${ }^{* *} 0.549$ & $14(87.5)$ & ${ }^{* *} 1.0$ \\
\hline Mujeres & $11(40.7)$ & $9(81.8)$ & & $9(81.8)$ & \\
\hline \multicolumn{6}{|l|}{ Procedencia } \\
\hline Urbano & $27(100)$ & $24(88.9)$ & & $23(85.2)$ & \\
\hline \multicolumn{6}{|l|}{ Estrato } \\
\hline Alto & $15(55.6)$ & $15(100)$ & ${ }^{* *} 0.075$ & $14(93.3)$ & ${ }^{* *} 0.294$ \\
\hline Medio & $12(44.4)$ & $9(75)$ & & $9(75)$ & \\
\hline
\end{tabular}

normalidad o la presencia de Hipoacusia Neurosensorial Grado I, II, III; se encontró pérdida auditiva en oído derecho del $88,9 \%$ (24) y en oído izquierdo del $85,2 \%$ (23). Los hombres evidenciaron perdida en oído derecho del $97,8 \%$ (15) y los de estrato alto el $100 \%(15)$ registraron la pérdida en este mismo oído. No se observaron asociaciones estadísticamente significativas de acuerdo a lo establecido en la escala Larsen modificado en oído derecho e izquierdo para ninguna de estas variables (Tabla 2).

Variables clínicas Al analizar la población evaluada, el 77,8\% (21) reportó tener dificultad para oír una conversación, el 93\% (25) no ha sufrido de vértigo; el $74 \%$ (20) siente o ha sentido tinitus, el $88,9 \%$ (24) no ha sufrido trauma en la cabeza, el $100 \%$ (27) no le han practicado cirugía de oídos, cabeza y se exponen 6 horas diarias al ruido de la pieza de alta velocidad. El 59,3\% (16) no sufre de otalgia y el $85,2 \%(23)$ no presenta hipertensión arterial. No se observaron asociaciones estadísticamente significativas de acuerdo a lo establecido en la escala de ELI en ambos oídos para ninguna de estas variables (Tabla 3).

De los odontólogos evaluados que refirieron oír con dificultad una conversación, el 66,7\% (14) evidenciaron pérdida auditiva en el oído izquierdo, quienes manifestaron presentar tinitus tuvieron alteración en el oído izquierdo del $70 \%$ (14); aque- 
llos que sufrían de otalgia su disminución fue en el mismo oído del $82 \%$ (9) y quienes sufrían de hipertensión se encontraron con alteración en ambos oídos en un $75 \%$ (3). No se observaron asociaciones estadísticamente significativas de acuerdo a lo establecido en la escala ELI en oído derecho e izquierdo para ninguna de estas variables (Tabla 3).
El 90,5\% (19) de los odontólogos evaluados que refirieron dificultad para oír una conversación presentaron perdida auditiva en el oído derecho, quienes reportaron padecer de tinitus o zumbidos en los oídos evidenciaron pérdida auditiva bilateral del $90 \%$ (18), aquellos que manifestaron padecer o haber padecido otalgia tuvieron alteración bilateral del 90,9\% (10) y quienes presentaron hipertensión arterial su disminución fue bilateral en el $100 \%$ (4).

Tabla 3. Descripción de las variables clínicas analizadas en forma global y según la calificación de Trauma Acústico mediante la Escala ELI.

\begin{tabular}{|c|c|c|c|c|c|}
\hline \multirow{4}{*}{ VARIABLE } & \multicolumn{5}{|c|}{ TRAUMA ACÚSTICO (ELI) } \\
\hline & GLOBAL & DERECHO & & IZQUIERDO & \\
\hline & & PERDIDA & $\mathbf{P}$ & PERDIDA & $\mathbf{P}$ \\
\hline & Frec. (\%) & Frec. (\%) & & Frec. (\%) & \\
\hline \multicolumn{6}{|l|}{ Oye con dificultad } \\
\hline No & $6(22.2)$ & $2(33.3)$ & ${ }^{* *} 0.357$ & $3(50.0)$ & ${ }^{* *} 0.638$ \\
\hline $\mathrm{Si}$ & $21(77.8)$ & $13(61.9)$ & & $14(66.7)$ & \\
\hline \multicolumn{6}{|l|}{ Tinitus } \\
\hline No & $7(25.9)$ & $2(28.6)$ & ${ }^{* *} 0.185$ & $3(43.0)$ & ${ }^{* *} 0.365$ \\
\hline $\mathrm{Si}$ & $20(74.0)$ & $13(65.0)$ & & $14(70.0)$ & \\
\hline \multicolumn{6}{|l|}{ Otalgia } \\
\hline No & $16(59.3)$ & $8(50.0)$ & 0.696 & $8(50.0)$ & ${ }^{* *} 0.124$ \\
\hline $\mathrm{Si}$ & $11(40.7)$ & $7(63.6)$ & & $9(82.0)$ & \\
\hline \multicolumn{6}{|l|}{ Hipertensión } \\
\hline No & $23(85.2)$ & $12(52.2)$ & 0.605 & $14(61.0)$ & ${ }^{* *} 1.000$ \\
\hline $\mathrm{Si}$ & $4(14.8)$ & $3(75.0)$ & & $3(75.0)$ & \\
\hline
\end{tabular}

Tabla 4. Descripción de las variables clínicas analizadas en forma global y según el Umbral Auditivo Evaluado mediante Escala LARSEN.

\begin{tabular}{|c|c|c|c|c|c|}
\hline \multirow{4}{*}{ VARIABLE } & \multicolumn{5}{|c|}{ UMBRAL AUDITIVO (LARSEN) } \\
\hline & \multirow{3}{*}{$\begin{array}{l}\text { GLOBAL } \\
\text { Frec. (\%) }\end{array}$} & \multirow{3}{*}{$\begin{array}{l}\text { DERECHO } \\
\text { PERDIDA } \\
\text { Frec. (\%) }\end{array}$} & \multicolumn{3}{|c|}{ IZQUIERDO } \\
\hline & & & $\mathbf{P}$ & PERDIDA & $\mathbf{P}$ \\
\hline & & & & Frec. (\%) & \\
\hline \multicolumn{6}{|l|}{ Oye con dificultad } \\
\hline No & $6(22.2)$ & $5(83.3)$ & ${ }^{* *} 0.545$ & $5(83.3)$ & **1.000 \\
\hline $\mathrm{Si}$ & $21(77.8)$ & $19(90.5)$ & & $18(85.7)$ & \\
\hline \multicolumn{6}{|l|}{ Tinitus } \\
\hline No & $7(25.9)$ & $6(85.7)$ & ${ }^{* *} 1.000$ & $1(71.4)$ & ${ }^{* *} 0.269$ \\
\hline $\mathrm{Si}$ & $20(74.0)$ & $18(90.0)$ & & $18(90.0)$ & \\
\hline \multicolumn{6}{|l|}{ Otalgia } \\
\hline No & $16(59.3)$ & $14(87.5)$ & ${ }^{* *} 1.000$ & $13(81.3)$ & ${ }^{* *} 0.624$ \\
\hline $\mathrm{Si}$ & $11(40.7)$ & $10(90.0)$ & & $10(90.9)$ & \\
\hline \multicolumn{6}{|l|}{ Hipertensión } \\
\hline No & $23(85.2)$ & $20(86.9)$ & ${ }^{* *} 1.000$ & $19(83.0)$ & **1.000 \\
\hline $\mathrm{Si}$ & $4(14.8)$ & $4(100)$ & & $4(100)$ & \\
\hline$\alpha=0,05$ & ${ }^{*}$ Test de Student & & exacto de & & \\
\hline 100 & & & & & \\
\hline
\end{tabular}


No se observaron asociaciones estadísticamente significativas de acuerdo a lo establecido en la escala Larsen modificado en los dos oídos para ninguna de estas variables (Tabla 4).

\section{DISCUSIÓN}

El presente estudio se dirigió a odontólogos de las clínicas de la Universidad Santo Tomás y en los que se determinó pérdida auditiva inducida por ruido con una prevalencia del 62,9\% para el oído izquierdo establecida por la calificación de la escala ELI y del $88,9 \%$ en el oído derecho según valoración emitida por la escala de calificación Larsen.

La Pérdida Auditiva Inducida por Ruido (PAIR) es de carácter neurosensorial irreversible causada por la exposición prolongada durante varios años a ruido intenso, esta pérdida aunque usualmente no produce dolor, causa interferencia en la comunicación además, puede afectar sustancialmente la integración social y la calidad de vida de las personas que la padecen. ${ }^{15}$

$\mathrm{Al}$ entrar a evaluar en este estudio la pérdida auditiva ocasionada por ruido en los odontólogos se tomó como base la calificación dada por las escalas ELI y Larsen modificado de diagnóstico ocupacional de acuerdo a los parámetros establecidos para dichos indicadores; aspecto que podría considerarse como una fortaleza del presente trabajo. ${ }^{7,16}$

Según la escala ELI la pérdida auditiva o (trauma acústico) de los odontólogos examinados presentó perdida auditiva del $55,6 \%$ (15) para el oído derecho y el $62,9 \%$ (17) para el oído izquierdo. Se observó que las mujeres tuvieron mayor pérdida auditiva en los dos oídos $63,6 \%$ correspondiente al oído derecho y el $72,7 \%$ para el oído izquierdo. Estos resultados coincidirían con los realizados por Forman Franco 1978, quien realizó audiometrías a 70 odontólogos de diferentes edades, especialidades y años de profesión y no encontró diferencia estadística cuando comparó los resultados de los niveles de audición ajustados con la edad de la población en general, pero si encontró alteración en las frecuencias de $4000 \mathrm{~Hz}$ y 6000 Hz por exposición continua durante 7 años. ${ }^{17}$

El National Institute for Ocupational Safety and Health (NIOSH) estableció que $25 \mathrm{~dB}$ es el máximo parámetro aceptable para empezar a hablar de disminución auditiva; además consideró que la frecuencia de $4000 \mathrm{~Hz}$ es tomada como estándar de daño auditivo si refleja alguna alteración en el limite de exposición para ruidos intensos y continuos. Este resultado podría explicarse como el inicio del proceso de alteración auditiva púes según los autores se determinó que la primera frecuencia que se altera por exposición a ruidos altos, repetitivos y durante largo tiempo es la de $4000 \mathrm{~Hz}{ }^{16}$

Al confrontar con los resultados encontrados en la escala ELI, las mujeres presentaron pérdida auditiva bilateral con inclinación hacia el oído izquierdo; con la calificación Larsen los hombres presentaron alteración auditiva bilateral con predominio oído derecho. Esta situación sustenta el progreso de la pérdida auditiva en los hombres, pues ya no solamente se encuentra involucrada la frecuencia de $4000 \mathrm{~Hz}$ sino las otras frecuencias agudas vecinas y algunas frecuencias conversacionales. Por otra parte, los resultados parecen indicar que las mujeres están en la etapa inicial de la alteración, debido muy posiblemente a que ellas tienen menos horas de trabajo a lo largo de su vida con reducción de la exposición al ruido, y se soporta en el hecho, en el que eventualmente deben asumir compromisos adicionales como son el cuidado de los hijos, el hogar, las licencias de maternidad, entre otros. Esta hipótesis queda abierta para futuras investigaciones que entren a evaluar estos aspectos dentro de la población de los odontólogos.

Merluzzi (1981) sugirió que la exposición al ruido diariamente con periodos repetidos durante varios años acumula efectos que producen un deterioro lento y progresivo inicialmente imperceptible de tipo neurosensorial que no necesariamente es bilateral, el autor define que si pasa de los $40 \mathrm{~dB}$ la disminución del umbral en las frecuencias de 4000 , 6000 y $3000 \mathrm{~Hz}$ va a evolucionar lentamente a las frecuencias de 8000 - 2000 - 1000,500 y $250 \mathrm{~Hz}$ éstas ultimas demoran mas tiempo en llegar a comprometerse. ${ }^{18}$

Al analizar cada escala de evaluación ocupacional se encontraron similitudes bilaterales en la pérdida auditiva de los odontólogos en lo que se refiere a la presencia de tinitus en el $74 \%$. Otro factor tenido en cuenta fue la manifestación de otalgia que evidenció el 90,9\% no arrojó valores mayores de incidencia de pérdida auditiva. La dificultad para oír una conversación reportó prevalencia en el $77,8 \%$. Lo anterior corrobora lo descrito por de De Melio Angela (1999) en donde sustenta que el zumbido también llamado acúfenos o tinitus y la otalgia son características subjetivas de disminución auditiva, las cuales constituyen una queja frecuente de trabajadores con lesiones auditivas inducidas por ruido. ${ }^{17}$

El odontólogo suele presentar alteraciones que se relacionan con los problemas auditivos, falta 
de comprensión del lenguaje o de sonidos medio ambientales y las no auditivas influenciadas en lo psicosocial como estrés, ansiedad, manía, depresión, nauseas, inestabilidad, jaquecas, nerviosismo, hostilidad, intolerancia, agresividad, aislamiento social y disminución de la tendencia natural hacia la ayuda mutua ${ }^{5}$, aislamiento que comprometen las relaciones del individuo en la familia, en el trabajo, afecta el desempeño laboral cada día.

Cooperman (1965) encontró que el uso prolongado de la pieza de alta puede elevar la pérdida auditiva parcial o permanente, se afecta la comunicación y cuando el ruido se eleva pasa inadvertido; por otro lado Kil, en 1981, criticó la hipótesis de la relación de pérdida auditiva y la edad; en su estudio encontró que los niveles de ruido de la turbina que oscilaban entre $70 \mathrm{a} 92 \mathrm{~dB}$, podrían afectarse por factores como son: la distancia del oído del profesional con respecto a la mano que manipula a la pieza de alta velocidad, más la posición del oído con relación a la fuente, más la posición del instrumento en la cavidad oral, determinó un promedio de 15 a 45 minutos para el uso de instrumento en una jornada de ocho (8) horas. ${ }^{5}$

Es importante resaltar que en este estudio quienes no reportaron padecer de hipertensión $(85,2 \%)$ tuvieron pérdida auditiva del $52,2 \%$ en oído derecho y del $61,0 \%$ en oído izquierdo en la escala ELI y en la escala Larsen oído derecho $86,9 \%$ y oído izquierdo $83 \%$ lo cual no puede relacionarse como factor desencadenante a la exposición a ruido. Durante años se han realizado estudios con el objetivo de analizar la posible relación entre la exposición a ruido y el desarrollo de hipertensión arterial (HTA), con resultados diversos, así pues mientras una parte de los autores encuentran una asociación entre estas, otros no hallan una relación significativa. Queda la inquietud para próximas investigaciones con respecto a los datos arrojados por este trabajo en cuanto a la HTA y la pérdida auditiva como alternativa que permita estudiar más a fondo acerca del tema.

Santana, en 1995, postula una hipótesis de la relación HTA con exposición al ruido ocupacional en donde se encontró que los niveles tensiónales sistólico y diastólico eran mas elevados en trabajadores industriales con hipoacusia generada por ruido, comparándose con un grupo de personas con la misma edad y sin pérdida auditiva. ${ }^{19}$

Otros estudios como el de Lang (1992) indican la relación del ruido excesivo con HTA y la reacción del sistema circulatorio y el estrés, en donde se evidencia aumento de las catecolaminas en la corriente sanguínea, produciendo vasoconstricción periférica y elevando así la frecuencia cardiaca asociada a un aumento de la presión arterial temporal. ${ }^{19}$

Es importante anotar que en el presente estudio se incluyó además la aplicación de la escala SAL (Speech Average Loss), ${ }^{20}$ para determinar el grado de audición que tiene el trabajador en las frecuencias conversacionales; el cual no arrojo resultados relevantes en la población evaluada por lo cual no se tuvo en cuenta en el momento de realizar las tablas y representar el análisis de los resultados.

Se consideró una limitante del estudio el tamaño de la muestra que no es, realmente, representativo y dificultó el hallazgo de los factores que implicaran una asociación estadísticamente significativa de la alteración auditiva producida por el ruido ocasionado durante la jornada laboral y el uso de la pieza de alta velocidad en los odontólogos.

Se plantea la opción de continuar con el estudio con un tamaño de muestra adecuado que permita indagar más al respecto; corroborar los resultados aquí obtenidos y ampliar los aspectos no contemplados, como podrían ser las alteraciones sistémicas, la realización de una sonometría, con el fin de determinar el valor exacto en decibeles generados en las clínicas para valorar la exposición provocada por el ambiente laboral tomando como parámetro de evaluación la actividad simultanea de los implementos generadores de ruido intenso según la jornada o especialidad que se este realizando dentro de las clínicas odontológicas. Así como llegar a considerar intervenciones orientadas a promover actitudes personales que permitan promover la salud y prevenir las patologías auditivas previas en los odontólogos.

\section{CONCLUSIONES}

- Los odontólogos, docentes de las clínicas odontológicas de la Universidad Santo Tomás, evidenciaron alteraciones auditivas según las escalas de calificación ocupacional ELI y LARSEN modificado.

- Con la medición de la Escala ELI se encontró trauma acústico en los odontólogos especialmente en las mujeres quienes presentaron disminución bilateral en la frecuencia de $4000 \mathrm{~Hz}$. El oído izquierdo a nivel global fue reportado con mayor afectación en el $62,9 \%$ lo cual evidencia que ellas se encuentran en el inicio de la alteración auditiva ocasionada por la exposición continua a los ruidos provocados dentro de su ambiente laboral. 
- El umbral auditivo de los hombres presentó mayor alteración bilateral en las frecuencias agudas y algunas conversacionales. El oído derecho a nivel global reportó mas alteración en el $88,9 \%$ lo cual argumenta que la disminución auditiva abarca mas frecuencias dentro de la escala de medición Larsen Modificado, por lo que se determinó que el proceso de evolución es mas notorio que en el sexo femenino.

- Los odontólogos de las clínicas efectivamente evidenciaron la presencia de síntomas relacionados con pérdida auditiva como son la presencia de tinitus o zumbidos durante su cotidianidad.

- La hipertensión arterial no representó ser un indicador que esté directamente relacionado con la exposición al ruido en el ambiente laboral, ni correspondió ser un factor de riesgo para padecer pérdida auditiva.

\section{RECOMENDACIONES}

- Es necesario reconocer la importancia del uso de los protectores auditivos dentro de las clínicas odontológicas pues es el único aditamento de fácil uso, manejo y acceso que puede proteger de los decibeles generados por los instrumentos de uso odontológico cuyo funcionamiento causa fatiga, adaptación y pérdida auditiva con el tiempo en los profesionales. Esta situación no es ajena al odontólogo pero aún no hay la suficiente conciencia y educación al respecto para que sea utilizada como medio de prevención y así evitar una futura enfermedad profesional.

- Realizar un estudio con mayor población de odontólogos cuya actividad exclusiva sea en su consultorio y que permanezcan más de 6 horas durante el día expuestos al ruido del ambiente de trabajo.

- Tomando como base este estudio ampliar la información acerca de las fuentes que generan ruido en las clínicas odontológicas de la USTA, midiendo la intensidad de los decibeles producidos durante el funcionamiento de todas las unidades que hay en cada modulo al mismo tiempo a través de una sonometria que determine cuanto es el máximo de decibeles que se alcanza a producir en una clínica regularmente.

- Identificar si el ruido de la clínica de pediatría es similar o superior al de la clínica integral para determinar si existe diferencia en los decibeles producidos.
- Hacer procesos de monitoreo y programas en promoción y prevención.

\section{BIBLIOGRAFÍA}

1. Azevedo A. Actualización del zumbido. Revista Brasilera de Otorrinolaringología 2004; 70;

2. Cavalli Regina CM, Morata Thais C, Marques Jair M. Hearing loss prevention programs control of Curitiba (HLPP). Rev Bras Otorrinolaringol [en línea] 2004 June [fecha de acceso: febrero de 2007]; 70 (3): 368 - 377. URL disponible en: http://www.scielo.br/scielo.php?script $=$ sci_arttext\& pid $=$ S003472992004000300013\&lng $=$ en\&nrm $=$ iso. doi: 10.1590/S0034-72992004000300013

3. Corzo G. El ruido en el ámbito laboral. URL disponible en: www.ergotema.com.

4. De Melio A. Alarma al ruído ocupacional. CEFAC Porto Alegre 1999.

5. Montes Ribeiro De Souza HM. Pérdida auditiva en odontólogos por ruido de la pieza de alta. Escuela Nacional Salud Pública - Fundación Oswaldo Cruz. Río de Janeiro, 1998.

6. Skock TA. Vigilancia de la exposición de ruido en el lugar de trabajo en Dinamarca. Occup Environ Med 2002: 59: $636-639$.

7. Lopez AC. Estudio de la alteración temporal del umbral auditivo en trabajadores de industria. CEFA, Sao Paulo, Brasil.

8. Grimmes DA, Schultz K. An overview of clinical research. The lay of the land. Lancet 2002; 359: $57-61$.

9. Microsoft Excel 5.0, Microsoft Corporation 1997.

10. CDC-OMS. Epi-Inf 6 Versión 6.04a Nov 1996.

11. Stata Corp 2003. Stata Statistical Software. Release $9.1 \mathrm{Col}-$ lege station, T.X: Stata Corporation.

12. Pagano M, Gauvreau K. Principles of Biostatistics. Duxbury Press. Belmont p. 35 - 44, 257 - 263.

13. Norman GR, Streiner DL. Bioestadística. Mosby/Doyma Libros. Madrid. 1996 p 129-147.

14. República de Colombia, Ministerio de Salud. Resolución No. 008430 del 4 de octubre de 1993.

15. Sliwinska-Kowalska M, Jedlinska U. Prolonged exposure to industrial noise: cochlear pathology does not correlate with the degree of permanent threshold shift, but is related to duration of exposure. J Occup Health 1998; 40: 123 $-131$.

16. Warner AF. Exposición al ruido ocupacional nuevos criterios y estándares internacionales. Salud Pública 1998; 37.

17. Pimentel de Souza F. Laboratório de Psicofisiologia, ICBUFMG, Belo Horizonte, Brasil. Efeito do ruído no homem dormindo e acordado. URL disponible en: http://www.icb. ufmg.br/lpf/pimentel,sobrac2000.html Belo Horizonte, 2000.

18. Vieira MR. Rol de fonoaudiólogo en la prevención de la pérdida auditiva inducida por ruido. CEFAC, Reafe 2000.

19. Santana SV. Exposición ocupacional al ruido y la hipertensión arterial. Salud Pública 1995; 29.

20. Vilas J. Valoración del trauma acústico. Centro de Investigación Asistencia Técnica - Barcelona. URL disponible en: http://www.mtas.es/insht/ntp/ntp_136.htm 